\title{
Adaptación y validación del cuestionario sentimiento de autoeficacia en una muestra de profesores chilenos
}

\author{
Adaptation and Validation of the Self efficacy Sense \\ Questionnaire in a Sample of Chilean Teachers
}

Recibido: 16 de octubre de 2014| Aceptado: 11 de febrero de 2016

\author{
CARMen Gloria Covarrubias ApablaZA ** \\ Universidad de Talca, Chile \\ Michelle Celinda Mendoza LiRA ${ }^{* * *}$ \\ Universidad Autónoma de Chile, Chile
}

doi : 10.11144/Javeriana.upsy15-2.avcs

Para citar este artículo: Covarrubias-Apablaza, C.G., \& Mendoza-Lira, M. C. (2016). Adaptación y validación del cuestionario sentimiento de autoeficacia en una muestra de profesores chilenos. Universitas Psychologica, 15(2), 97-108. http://dx.doi. org/10.11144/Javeriana.upsy15-2.avcs

* Artículo de investigación científica y tecnológica.

** Profesora del Programa de Formación Fundamental dependiente, Vicerrectoría de Pregrado. Correo electrónico: cacovarrubias@utalca.cl

*** Profesora e Investigadora en la Facultad de Educación. Correo electrónico: mmendozal@uautonoma.cl

\section{RES UMEN}

Este trabajo tiene el propósito de exponer el proceso de adaptación y validación del cuestionario Teacher Self-Efficacy Scale (Tschannen-Moran \& Woolfolk, 2001) en una muestra de 544 profesores chilenos. Para ello, se utilizó el procedimiento de traducción inversa. Por su parte, para la validez de constructo se aplicó, en primer lugar, la técnica estadística de análisis factorial exploratorio, la cual arrojó un factor más para la versión de tres factores del instrumento original. En segundo lugar, se realizó un análisis factorial confirmatorio que corroboró la solución de cuatro dimensiones con adecuados índices de ajuste. Finalmente, se analizó la consistencia interna a través del coeficiente de Alpha de Cronbach, evidenciando índices satisfactorios tanto en cada uno de los factores como en la totalidad del instrumento. Todos estos hallazgos permiten afirmar que la versión adaptada del cuestionario es válida, fiable y explicativa de la teoría de la autoeficacia para la realidad de los docentes chilenos.

Palabras clave

autoeficacia; adaptación; validación; educación; profesores

\section{A B S T R A C T}

The purpose of this paper is to show the adaptation and validation process of the Teacher Self-Efficacy Scale questionnaire (Tschannen-Moran $\&$ Woolfolk, 2001) in a sample selection of 544 Chilean teachers. This was done using the back translation procedure. For construct validity, the exploratory factor analysis statistic method was applied, which resulted in one additional factor to the three, which were in the original instrument. Secondly, a confirmatory factor analysis was carried out. The latter corroborated the four-dimension solution with appropriate index adjustments. Finally, the internal consistency was analyzed by using the Cronbach's alpha coefficient, which showed satisfactory rates for each factor and the entire instrument. All these findings support the claim that the adapted version of the questionnaire is valid, reliable and explanatory of the self-efficacy theory for the reality of Chilean teachers.

Keywords

self-efficacy; adaptation; validation; education; teachers 


\section{Introducción}

La teoría de autoeficacia ha sido motivo de estudio en diversos ámbitos del funcionamiento humano como la política, la economía, el deporte, la salud y la educación. En esta última área, conocer los sentimientos que tienen los profesores acerca de su autoeficacia arroja información relevante de sus desempeños pedagógicos, pues su compleja influencia resulta indiscutible en las acciones que emprenden tanto en la generación de aprendizajes en los estudiantes como en la comunidad escolar.

Efectivamente, las investigaciones de dichos sentimientos constituyen un área de información valiosa acerca de cuán capaces se sienten para ejecutar las tareas de enseñanza y promover el aprendizaje en todos los estudiantes. En este sentido, resultados de innumerables estudios avalan que sentimientos de autoeficacia positivos fomentan el uso de estrategias de enseñanza y aprendizaje variadas, la adaptación de la enseñanza a las singularidades educativas de los aprendices, el manejo eficaz de los conflictos de aula y la promoción de la participación en los estudiantes, entre otros aspectos.

Dicho lo anterior, y debido a la relevancia de este tema en el sector educativo, la investigación que se presenta a continuación, tiene el propósito de describir los sentimientos de autoeficacia en una muestra de 544 profesores chilenos. Para ello, se ha realizado la traducción y la adaptación del cuestionario Teacher Sense of Efficacy Scale (TSES) de Tschannen-Moran y Woolfolk (2001), cuyo proceso de validación ha arrojado índices interesantes tanto para su estructura factorial como para su consistencia interna.

Hay que mencionar, además, que las opiniones vertidas por esta muestra de profesores han revelado que el género no representa una variable de cambio en los sentimientos de autoeficacia docente; así como tampoco lo son los niveles de enseñanza infantil, primario y/o secundario donde estos se desempeñan. Por el contrario, la experiencia docente y la titularidad del centro — pública, concertada y/o privada_sí han reportado diferencias estadísticamente significativas. Ahora bien, con el propósito de comprobar el efecto de las variables indepen- dientes sobre las variables dependientes se llevó a cabo un análisis conjunto de todas ellas, el cual no ha arrojado diferencias significativas.

\section{Marco teórico}

\section{Teoría de la autoeficacia}

La teoría de la autoeficacia ha pasado por distintas etapas conceptuales y metodológicas. Desde sus comienzos hasta la actualidad, esta teoría ha intentado demostrar cómo los aspectos cognitivos, conductuales, contextuales y afectivos de las personas están condicionados por la autoeficacia. Albert Bandura (1977), su máximo impulsor, preocupado por elevar a ciencia la psicología y validar sus postulados, buscó un método eficaz que resolviera problemas humanos.

Para lograr dicho cometido, dio forma y consistencia a su teoría, cuyo eje central, el pensamiento autorreferente, actúa mediando la conducta y la motivación en las personas. Por esta razón, los individuos no tienen un funcionamiento autónomo ni tampoco sus comportamientos están totalmente determinados por factores situacionales. Así pues, las personas son producto de la interacción recíproca entre determinantes personales, comportamentales y ambientales.

De igual manera, destacó que su teoría albergaba la presencia de dos tipos de expectativas: una de eficacia y otra de resultados. Las primeras han sido definidas como el convencimiento de que una ejecución se realice con éxito y, las segundas, como la estimación de los resultados a conseguir una vez realizada la ejecución (Bandura, 1999).

No obstante, Bandura (1987), consciente de que el poder de una teoría psicológica reside en la capacidad de definición, predicción y descubrimiento de factores que regulan interrelacionadamente la conducta humana, propuso mecanismos, fuentes y procesos implicados en la autoeficacia. En lo referido a los mecanismos, la autoeficacia opera según la selección de conductas; el esfuerzo y la persistencia; los pensamientos y las reacciones emocionales; así como la producción y predicción de la conducta. Aún más, el sentimiento de autoeficacia puede ser 
desarrollado a través de cuatro fuentes: las experiencias de dominio, las experiencias vicarias, la persuasión social y los estados psicológicos y emocionales. Incluso, alberga la capacidad de activar procesos cognitivos, motivacionales, afectivos y selectivos.

Por último, el impacto de lo antes dicho marca profundas diferencias en las personas con un fuerte o un débil sentimiento de autoeficacia. Las primeras poseen un interés intrínseco que las hace esforzarse y perseverar pese a las dificultades; son capaces de visualizar los problemas como desafíos, oportunidades de aprendizaje y, por ende, de progreso en sus habilidades. Por su parte, las segundas carecen de compromiso; flaquean rápidamente ante las adversidades; evitan tareas complejas; huyen de los problemas y dejan de creer en sí mismas.

\section{Autoeficacia y profesorado}

Para comprender la teoría de autoeficacia en el profesorado, resulta imprescindible exponer las dos perspectivas que han impulsado su estudio: una desde Julian Rotter y otra desde Albert Bandura.

Bajo la perspectiva de la teoría del aprendizaje social de Rotter (1966), surgió la primera línea de investigación sobre la eficacia del profesor. Conviene aclarar que Rotter nunca se refirió al constructo de autoeficacia como tal, sino que puso los cimientos sobre los cuales Bandura, años más tarde, basaría su propuesta (Browers \& Tomic, 2003). Los estudios derivados de esta perspectiva consideraron que la eficacia del profesor, junto con albergar una expectativa generalizada en torno a las consecuencias internas o externas de las conductas, constituía un rasgo relativamente estable acerca de las capacidades para ejercer control sobre sus conductas pedagógicas.

Un segundo enfoque para el estudio de autoeficacia del profesor provino de la teoría sociocognitiva de Bandura. Este psicólogo afirma que la autoeficacia está relacionada con las capacidades personales para identificar las oportunidades que ofrece el entorno e interpretar los obstáculos que se perciben. De ahí que la autoeficacia no constituya un rasgo estable de la personalidad, por el contrario, tiene que ver con un juicio de capacidad donde "no se es capaz para todo, ni a los mismos niveles" (Garrido, 2000, p. 13).

A juicio de otros expertos, la eficacia personal ejerce una poderosa influencia en los niveles de realización o logro de las personas (Pajares, 2006), mientras que Tschannen-Moran y Johnson (2011) sostienen que la autoeficacia comporta una evaluación de las capacidades reales para lograr un rendimiento adecuado en un ámbito de funcionamiento específico. De este modo, la eficacia del profesor constituye un tipo de autoeficacia que contempla una evaluación de las propias capacidades para alcanzar un nivel adecuado de rendimiento en una tarea y un entorno específico. No obstante, para alcanzar un desempeño óptimo, los docentes deben manejarse en dos niveles de pensamiento: por un lado, sentirse capaces y, por otro, juzgarse capaces (Bandura, 1987; Prieto, 2007).

Junto con ello, Tschannen-Moran y Woolfolk (2001) enfatizan en los sentimientos de autoeficacia como juicios de capacidad docente para enseñar y suscitar aprendizaje en determinadas y variadas circunstancias. Por su parte, recientes aportaciones surgen de la opinión de Ross y Bruce (2007), quienes conciben el sentimiento de autoeficacia del profesor como una expectativa que promueve el aprendizaje en los estudiantes. Otros consideran que la autoeficacia del profesor es la capacidad para realizar con éxito tareas específicas de enseñanza (Dellinger, Bobbet, Olivier, \& Ellet, 2008), mientras que Pas, Bradshaw y Hershfeldt (2012) opinan que este sentimiento está referido a la capacidad de los profesores para crear un ambiente adecuado para el aprendizaje.

Ahora bien, es importante recordar que el sentimiento de autoeficacia en el profesorado también se ve influenciado por el contexto del aula, la escuela y el entorno; además, por los estudiantes, los colegas, la dirección y la comunidad. Los profesores son líderes de un escenario de aprendizaje y a la vez empleados de una organización, ejecutan sus quehaceres pedagógicos cotidianos, socializan con el resto de la organización y contribuyen con la sociedad (Bueno, 2004; Friedman \& Kass, 2002), por ello, dichos sentimientos deben ser una muestra clara de que tienen las 
capacidades para ejecutar sus tareas con lo mejor de sus competencias cognitivas, afectivas y actitudinales.

\section{Sentimiento de autoeficacia en profesores chilenos}

Las escasas investigaciones sobre el sentimiento de autoeficacia en los profesores chilenos resultan preocupantes por el impacto del constructo en sus prácticas pedagógicas. Como consecuencia de lo anterior, gran parte de las búsquedas efectuadas arrojan sugerencias de estudios, revisiones teóricas o incipientes investigaciones empíricas, focalizadas mayormente en futuros profesores y, en menor medida, en profesores en ejercicio.

Considerando lo mencionado anteriormente, Ávalos, Cavada, Pardo y Sotomayor (2010), incitan al desarrollo de estudios que permitan comprender la identidad profesional del docente a partir de los sentimientos de autoeficacia, ausentes en los lineamientos de política educativa y política docente. Algo semejante, postulan Solís et al. (2011) sobre la necesidad de abordar la formación práctica de los futuros profesores desde diversos puntos de vista, siendo uno de ellos el sentimiento de autoeficacia.

Pese a este vacío investigativo, resalta el estudio de Guerra (2008), el cual reveló que las profesoras obtienen mayores puntuaciones en sus sentimientos autoeficacia, mientras que los profesores de enseñanza secundaria que poseen más experiencia y que se desempeñan en centros públicos presentan los niveles más bajos de autoeficacia. Cabe destacar, además, la investigación de Araya, Taut, Santelices y Manzi (2011), que mediante un diseño cualitativo logran identificar que los profesores evaluados con mejor desempeño no solo mejoran su valoración profesional, sino que, además, aumentan sus sentimientos de autoeficacia.

Para finalizar, Ávalos y Sotomayor (2012), con una investigación de enfoque mixto, abordan la identidad profesional docente con base en cinco componentes, siendo uno de ellos el sentimiento de autoeficacia. No obstante, y aun cuando una proporción importante de profesores estiman sentirse satisfechos con sus capacidades pedagógicas, los profesores de secundaria evidencian un menor nivel que sus colegas de enseñanza primaria. Por otra parte, los profesores que dominan los contenidos disciplinarios y la gestión del aula demuestran un mayor sentimiento de autoeficacia, lo que deriva en un menor cansancio y en una mayor satisfacción por lo que hacen.

\section{Metodología}

\section{Objetivo}

El objetivo general de este estudio ha sido adaptar y validar la Escala de Sentimiento de Autoeficacia en el Profesor (Teacher Self Efficacy Scale, TSES) para una muestra de profesores chilenos.

\section{Diseño}

La investigación realizada ha empleado una metodología cuantitativa no experimental, de tipología

TABLA 1.

Características de la Muestra

\begin{tabular}{lccccc}
\hline \multicolumn{5}{c}{ Variables } \\
\hline \multirow{2}{*}{ Género } & Femenino & Masculino & & \\
\cline { 2 - 3 } Nivel enseñanza & $70.2 \%$ & $29.8 \%$ & & \\
& Infantil & Primaria & Secundaria & \\
Titularidad Centro & $9.2 \%$ & $52.2 \%$ & $38.6 \%$ & & \\
& Público & Concertado & Privado & & \\
Experiencia (años) & $40.4 \%$ & $48.2 \%$ & $11.4 \%$ & & +20 \\
& $0-5$ & $6-10$ & $11-15$ & $16-20$ & $42.5 \%$ \\
\hline
\end{tabular}

Fuente: elaboración propia 
descriptiva, transeccional, y psicométrica (Hernández, Fernández, \& Baptista, 2010).

\section{Muestra}

La muestra, compuesta por 544 profesores de las áreas urbanas de las provincias de Marga-Marga, Quillota y Valparaíso (Chile). La participación de los sujetos ha sido voluntaria, encontrándose todos ellos en situación laboral activa y en posesión de la correspondiente titulación.

\section{Procedimiento para la recolección de datos}

Para acceder a los centros educativos se contactó a sus administradores, con el fin de solicitar la autorización para llevar a cabo la investigación. Una vez conseguidas las correspondientes autorizaciones, se procedió a enviar a los profesores una carta de presentación y el cuestionario a cumplimentar.

\section{Instrumento}

El instrumento de medida seleccionado que mejor se adaptaba a las necesidades de esta investigación fue el Teachers Self Efficacy Scale (TSES) de Tschannen-Moran y Woolfolk (2001), en su versión larga. El instrumento consta de 24 ítems, puntuables en una escala de 1 a 9 (1= "nada", 9= "mucho"), que se dividen en 3 sub-escalas: a) "Eficacia para la implicación de los estudiantes", b) "Eficacia en las estrategias de enseñanza" y c) "Eficacia en el manejo de la clase”. Posteriormente, se llevó a cabo el proceso de traducción y adaptación del TSES a lengua española. El procedimiento utilizado para la traducción de la medida del inglés al español fue el de traducción inversa o back translation (Martínez, Moreno, \& Muñiz, 2005).

\section{Estudio piloto}

Los participantes del proceso de validación del instrumento fueron 262 profesores de diferentes centros educativos públicos, concertados y privados de los ayuntamientos de Valparaíso y Viña del Mar.
Respondieron al instrumento con una escala tipo Likert simplificada de 1 ("nada") a 5 ("mucho"). El cambio de escala responde a las necesidades del contexto esgrimidas por los expertos y por los participantes de una prueba prepiloto, de la que se concluyó que una escala con más opciones podría ocasionar confusiones.

\section{Resultados}

\section{Validez de constructo}

Los datos fueron analizados estadísticamente con los programas SPSS (v. 17) y AMOS (v. 5.0) para Windows. Las técnicas estadísticas seleccionadas que mejor se ajustaban a los datos para llevar a cabo la validación de constructo han sido el análisis factorial exploratorio, el análisis factorial confirmatorio y el índice de consistencia interna Alfa de Cronbach.

\section{Estudio 1: Análisis Factorial Exploratorio}

Como muestra la Tabla 2, la validez de constructo realizada con análisis factorial exploratorio arrojó, en los tres análisis factoriales, la presencia de cuatro factores. Pese a que los resultados no coinciden con la solución original del instrumento ( 3 factores con 24 ítems), es posible destacar el aumento sostenido de la varianza total explicada (desde el primer al tercer análisis). Junto con ello, hay que resaltar el logro de índices de consistencia interna bastante altos, lo que hace que el instrumento sea válido y fiable.

\section{Estudio 2: Análisis Factorial Confirmatorio}

La validez factorial de la Escala de Sentimiento de Autoeficacia en Profesores se comprobó mediante análisis factorial confirmatorio, empleando el programa AMOS 5.0. Con el propósito de confirmar la estructura factorial obtenida, se utilizó el método de estimación de máxima verosimilitud junto con el procedimiento bootstrapping para comprobar que los estimadores no estaban afectados por la falta de normalidad y, por tanto, eran robustos (Byrne, 2001). 
TABLA 2.

Análisis Factorial Exploratorio

\begin{tabular}{|c|c|c|c|}
\hline $\mathrm{AFC}$ & Factores Extraídos & $\begin{array}{l}\text { Varianza Total } \\
\text { Explicada }\end{array}$ & Ítems \\
\hline \multirow{5}{*}{ Primero } & Factor A: Eficacia en la implicación de los estudiantes. & $17.10 \%$ & 6 \\
\hline & Factor B: Eficacia en las estrategias de instrucción & $33.70 \%$ & 6 \\
\hline & Factor C: Eficacia en el manejo de clase & $48.32 \%$ & 6 \\
\hline & \multirow{2}{*}{ Factor D: C"Eficacia en la atención a la singularidad de los estudiantes. } & \multirow{2}{*}{$59.62 \%$} & 6 \\
\hline & & & Total 24 \\
\hline \multirow{5}{*}{ Segundo } & Factor A: Eficacia en la implicación de los estudiantes. & $19.15 \%$ & 4 \\
\hline & Factor B: Eficacia en las estrategias de instrucción & $34.97 \%$ & 6 \\
\hline & Factor C: Eficacia en el manejo de clase & $50.42 \%$ & 5 \\
\hline & \multirow{2}{*}{ Factor D: C"Eficacia en la atención a la singularidad de los estudiantes. } & \multirow{2}{*}{$62.74 \%$} & 4 \\
\hline & & & Total 19 \\
\hline \multirow{5}{*}{ Tercero } & Factor A: Eficacia en la implicación de los estudiantes. & $19.55 \%$ & 4 \\
\hline & Factor B: Eficacia en las estrategias de instrucción & $36.63 \%$ & 4 \\
\hline & Factor C: Eficacia en el manejo de clase & $53.43 \%$ & 5 \\
\hline & \multirow{2}{*}{ Factor D: C"Eficacia en la atención a la singularidad de los estudiantes. } & \multirow{2}{*}{$65.09 \%$} & 4 \\
\hline & & & Total 17 \\
\hline
\end{tabular}

Fuente: elaboración propia

Para evaluar la bondad de ajuste del modelo, se utilizaron diferentes índices: Chi cuadrado $\left(\chi^{2}\right)$ y también la ratio entre chi cuadrado y los grados de libertad $\left(\chi^{2} / g l\right)$, ya que el $\chi^{2}$ es muy sensible al tamaño muestral (Jöreskog \& Sörbom, 1996). Junto con dichos índices se emplearon el Comparative Fit Index (CFI), Incremental Fit Index (IFI), Root Mean Square Error of Approximation (RMSEA) y Standardized Root Mean Square Residual (SRMR).

Cabe destacar que para el indicador $2 / g l$ se suelen aceptar valores inferiores a 3 (SchermellehEngel, Moosbrugger, \& Müller, 2003), mientras que valores CFI e IFI superiores a 0.95 — junto con valores iguales o menores a 0.06 para RMSEA y 0.08 PARA SRMR — indican buen ajuste del modelo (Hu \& Bentler, 1999).
Como se puede advertir en la Tabla 3, los resultados del análisis factorial confirmatorio revelaron buenos índices de bondad de ajuste relativos al modelo de cuatro factores para la Escala "Sentimiento de Autoeficacia en Profesores", a saber: $\chi^{2}$ $=290.90, p=0.00 ; \chi^{2} / g l=1.36 ; \mathrm{CFI}=0.95 ; \mathrm{IFI}=$ $0.96 ; \mathrm{SRMR}=0.05 ; \mathrm{RMSEA}=0.05$.

(a) $\quad \chi^{2} /$ d.f.: chi-cuadrado dividido por los grados de libertad

(b) $\quad * * \chi^{2}: p<0.0001$

Estudio 3: Análisis de fiabilidad

La fiabilidad total del cuestionario para 17 ítems arrojó un alfa de Cronbach de 0.922 (Cronbach, 1951). En tanto, la fiabilidad por factor osciló entre 0.737 y 0.838 (véase Tabla 4).

TABLA 3

Índices de bondad de ajuste del modelo propuesto

\begin{tabular}{ccccccc}
\hline Modelo & $\chi^{2}$ & $\chi 2 / g l$ & CFI & IFI & SRMR & RMSEA \\
\hline Cuatro factores & $290.9^{* *}$ & 1.36 & 0.959 & 0.960 & 0.05 & 0.54 \\
\hline
\end{tabular}

Fuente: elaboración propia 
TABLA 4.

Confiabilidad de las dimensiones. Método Alfa de Cronbach

\begin{tabular}{lcc}
\hline \multicolumn{1}{c}{ Factores } & $\mathrm{N}^{\circ}$ Ítems & $\alpha$ \\
\hline A: Eficacia en la implicación de los estudiantes. & 4 & 0.737 \\
B: Eficacia en las estrategias de enseñanza y aprendizaje. & 4 & 0.830 \\
\hline C: Eficacia en el manejo de la clase. & 5 & 0.838 \\
D: Eficacia en la atención a la singularidad de los estudiantes. & 4 & 0.816 \\
\hline Total & 17 & 0.922 \\
\hline
\end{tabular}

Fuente: elaboración propia

\section{Discusión y consideraciones finales}

Los análisis estadísticos realizados para la validez de constructo del cuestionario "Sentimiento de Autoeficacia en el Profesor" revelaron resultados y diferencias interesantes en la estructura factorial —exploratoria y confirmatoria-y en los índices de fiabilidad.

En concreto, los tres análisis factoriales exploratorios aplicados a la propuesta traducida y adaptada del citado cuestionario arrojaron una disminución de 24 a 17 ítems y, a su vez, el aumento de 3 a 4 factores con respecto a la versión original. De esta manera, a la solución inicial de tres factores: "Eficacia en la Implicación de los estudiantes"; "Eficacia en las Estrategias de Enseñanza y Aprendizaje" y "Eficacia en el Manejo de la Clase", se sumó un cuarto factor enfocado en la "Eficacia en la Atención a la Singularidad de los Estudiantes". Todo esto coincide con reportes sobre la validación de este constructo, los cuales han arrojado un promedio fluctuante entre 3 y 6 factores. Incluso, los investigadores — pese al desacuerdo- sostienen y se inclinan por un modelo de cuatro factores, ya que tendría un mejor ajuste con la teoría de autoeficacia (Browers \& Tomic, 2003; Henson, 2001; Malinen, Savolainen, \& Xu, 2012).

Habría que señalar también un aumento en el porcentaje acumulado de varianza total de 59\% a $65 \%$, considerado como un resultado realista para Henson y Roberts, (2006), aun cuando sea alejado del $75 \%$ obtenido por los autores del instrumento (Tschannen-Moran \& Woolfolk, 2001). A pesar de estas diferencias, los resultados son coincidentes con los obtenidos por Johar y Badrasawi (2009), que mantuvieron los 24 ítems del cuestionario, pero con una estructura definida por cinco factores, que finalmente explicaron en un porcentaje acumulado de varianza de $62 \%$. Asimismo, concuerda con los primeros resultados de Wolters y Daughtery (2007), a quienes les fue revelado un cuarto factor, pero, intencionadamente, mantuvieron la solución original de tres factores, lo que arrojó un $66 \%$ de varianza explicada.

Por su parte, el análisis factorial confirmatorio reveló valores coherentes para el modelo de cuatro factores de la versión chilena del Teacher Self-Efficacy Scale, con ello es posible indicar que obtiene un buen ajuste y, por lo mismo, queda justificado teóricamente. Cabe mencionar que, para el contexto de profesores chilenos, el cuestionario permite evaluar sus sentimientos con respecto a sus capacidades para lograr la eficacia en involucrar a los estudiantes, manejar estrategias de enseñanza y aprendizaje, gestionar la clase y atender la singularidad del aula (Forsyth \& Carey, 1998). Pese a esto, dicha solución no resulta coincidente ni con los análisis factoriales confirmatorios de dos factores ni tampoco con la reevaluación del factor concerniente a la eficacia que tienen los profesores para manejar la clase, sugerida por Robert y Henson (2001).

Por otro lado, aún cuando es complejo comparar los índices de fiabilidad de dos estructuras factoriales distintas, sí es posible indicar que para el total del cuestionario se obtuvo un alto índice de consistencia interna ( $\alpha=0.92)$, vale decir, dos puntos menos con respecto a la versión original. Pese a ello, las mayores diferencias pueden apreciarse al desglosar la fiabilidad por cada factor del cuestio- 
nario, que para la versión chilena fluctuaron entre 0.73 y 0.83 , mientras que para la versión original oscilaron entre 0.87 y 0.90 (Tschannen-Moran \& Woolfolk, 2001).

Dicho esto, al cotejar ambas versiones del cuestionario (estadounidense y chilena) es posible identificar índices de Alfa de Cronbach un tanto más bajos para el factor de "Eficacia en la Implicación de los Estudiantes” (0.87 y 0.73 , respectivamente). Más aún, se han obtenido semejantes resultados en muestras de profesores turcos, con un $\alpha=0.82$ (Sari, Çeliköz, \& Seçer, 2009); griegos, con un $\alpha$ $=0.78$ (Poulou, 2007) y malayos, con un $\alpha=0.69$ (Johar \& Badrasawi, 2009). Sin embargo, atendiendo a las características y finalidad de este estudio, cabe resaltar que son ampliamente aceptados estadísticos de fiabilidad de - al menos- 0.70 (Prieto \& Delgado, 2010).

A raíz de los diferentes resultados obtenidos, diversos estudiosos en la materia, tales como Tsigilis, Koustelios, \& Grammatikopoulos (2010), han advertido que las diferencias culturales o contextuales en las representaciones, conceptos e ideas acerca de la enseñanza podrían estar jugando a favor de las diferencias obtenidas en los estudios de unos y otros. Asimismo, Ho y Hau (2004) han enfatizado que las culturas y las prácticas educativas pueden variar e influir en los desempeños y, por lo tanto, en la opinión que tienen los profesores acerca de sus sentimientos de autoeficacia. No obstante, Klassen et al. (2009) han concluido que, pese a las diferencias contextuales y educativas patentes en las diversas culturas, los sentimientos de autoeficacia en el profesorado parecen tener patrones similares entre ellas.

Desde otro prisma, los resultados de esta investigación podrían explicarse por la utilización de una escala Likert de 1 a 5 , en lugar de 0 a 9 puntos. No obstante, la utilización de una escala Likert simplificada ha sido defendida por la viabilidad para medir los sentimientos de autoeficacia en los profesores, la capacidad para responder a las demandas del entorno y la claridad al momento de puntuar las opiniones evitando, así, las controversias (Croasmun \& Ostrom, 2011; Maurer \& Pierce, 1998; Tagle, del Valle, \& Flores, 2012).
Por otra parte, desde una perspectiva teórica, la propuesta chilena del TSES sumó un cuarto factor que, de acuerdo con el enfoque de los ítems que lo componen, se denominó "Eficacia en la Atención a la Singularidad de los Estudiantes". Esta nueva dimensión ha sido concurrente en el planteamiento métrico de E.M Skaalvik y S. Skaalvik (2007), cuyo instrumento considera a uno de sus factores responsable de medir la autoeficacia que tienen los profesores noruegos para adaptar la enseñanza a las necesidades educativas de los estudiantes. De manera análoga, Chan (2008) ha construido una medida de autoeficacia para profesores chinos que ha destinado dos de seis subescalas, para evaluar la eficacia del profesor para enseñar a estudiantes altamente capaces y atender la diversidad en el aula.

En consecuencia, el instrumento utilizado ha revelado poseer la fiabilidad y validez suficiente, no solo para capturar un $8 \%$ de las respuestas falsas emitidas por los profesores, sino también para albergar la calidad adecuada para generar interpretaciones e inferencias de las opiniones vertidas. Junto a ello, la estructura factorial, no siendo idéntica a la propuesta por los autores del instrumento, ha evidenciado un porcentaje de varianza realista para explicar tanto la teoría como un factor concerniente a los sentimientos de autoeficacia docente para atender la singularidad de los estudiantes.

\section{Referencias}

Araya, C., Taut, S., Santelices, V., \& Manzi, J. (2011). Validez consecuencial del programa de asignación de excelencia pedagógica en Chile. Estudios Pedagógicos, 37(2), 25-42.

Ávalos, B., Cavada, P., Pardo, M., \& Sotomayor, C. (2010). La profesión docente: temas y discusiones en la literatura internacional. Estudios Pedagógicos, 36(1), 235-263.

Ávalos, B., \& Sotomayor, C. (2012). Cómo ven su identidad los docentes chilenos. Perspectiva Educacional, 51(1), 77-95.

Bandura, A. (1977). Self-efficacy: Toward a unifying theory of behavioral change. Psychological Review, 84(2), 191-215. 
Bandura, A. (1987). Pensamiento y acción. Fundamentos Sociales. Barcelona: Martínez Roca.

Bandura, A. (1999). Ejercicio de la eficacia personal y colectiva en sociedades cambiantes. En A. Bandura (Ed.), Autoeficacia. Cómo afrontamos los cambios de la sociedad actual (pp.19-54). Bilbao: Descleé De Brouwer.

Browers, A., \& Tomic, W. (2003). A test of the factorial validity of teacher efficacy scale. Research in Education, 69, 67-79.

Bueno, J. A. (2004). La motivación del alumno en el aula. Madrid: ICCE

Byrne, B. M. (2001). Structural equation modeling with AMOS: Basic concepts, applications and programming. Mahwah, NJ: Erlbaum.

Croasmun, J. T., \& Ostrom, L. (2011). Using Likert-type in the social sciences. Journal of Adult Education, 4(1), 19-22.

Cronbach, L. (1951). Coefficient alpha and the internal structure of tests. Psychometrika, 16(3), 297-334.

Chan, D. (2008). Dimensions of teacher self-efficacy among Chinese secondary school teachers in Hong Kong. Educational Psychology, 28(2), 181-194.

Dellinger, A. B., Bobbett, J. J., Olivier, D. F., \& Ellett, C. D. (2008). Measuring teachers' self-efficacy beliefs: development and use of the TEBS-Self. Teaching and Teacher Education, 24(2), 751-766.

Friedman, I., \& Kass, E. (2002). Teacher self-efficacy: a classroom-organization conceptualization. Teaching and Teacher Education, 18(6), 675-686.

Forsyth, A. D., \& Carey, M. P. (1998). Measuring selfefficacy in the context of HIV risk reduction: research challenges and recommendations. Health Psychology, 17(6), 559-568.

Garrido, E. (2000). Autoeficacia en el mundo laboral. Apuntes de Psicología, 18(1), 19-38.

Guerra, P. (2008). Creencias epistemológicas y de eficacia docente de profesores que postulan al programa de acreditación de excelencia pedagógica y su relación con las prácticas de aula. Tesis de Maestría. Santiago de Chile: Pontificia Universidad Católica de Chile. Recuperado de http://uc-cl. academia.edu/paulaguerra/Books/700783/Creencias_epistemologicas_y_de_eficacia_docente_de profesores_que_postulan_al_programa_de_acreditacion_de_excelencia_pedagogica_y_su_rela-
cion_con_las_practicas_de_aula._Tesis_de_Magister_en_Psicologia_Educacional_Pontificia_ Universidad_Catolica_de_Chilee

Henson, R. K. (2001, enero). Teacher self-efficacy: substantive implications and measurement dilemmas. Comunicación presentada at the Annual Meeting of the Educational Research Exchange, Texas.

Henson, R. K., \& Roberts, J. K. (2006). Use of exploratory factor analysis in published research: Common errors and some comment on improved practice. Educational and Psychological Measurement, 66(3), 393-416.

Hernández, R., Fernández, C., \& Baptista, P. (2010). Fundamentos de metodología de la investigación (2a. ed.). Madrid: McGraw-Hill.

Ho, I., \& Hau, K. (2004). Australian and Chinese teacher efficacy: similarities and differences in personal instruction, discipline, guidance efficacy and beliefs in external determinants. Teaching and Teacher Education, 20(3), 313-323.

Hu, L., \& Bentler, P. M. (1999). Cutoff criteria for fit indexes in covariance structure analysis: conventional criteria versus new alternatives. Structural Equation Modeling, 6, 1-55.

Johar, E., \& Badrasawi, K. (2009, June). The construct validation of teachers' efficacy scale in the Malaysian context. Paper presented at the $6^{\text {th }}$ International Postgraduate Research Colloquium, Bangkok, Thailand. Recuperado de http://bsris.swu.ac.th/ iprc/6th/9.pdf

Jöreskog, K. G., \& Sörbom, D. (1996). LISREL 8: User's reference guide. Chicago, IL: Scientific Software International.

Klassen, R. M., Bong, M., Usher, E. L., Chong, W., Huan, V. S., Wong, I., \& Georgiou, T. (2009). Exploring the validity of a teachers' self-efficacy scale in five countries. Contemporary Educational Psychology, 34(1), 67-76.

Malinen, O. P., Savolainen, H., \& Xu, J. (2012). Beijing in-service teachers' self-efficacy and attitudes towards inclusive education. Teaching and Teacher Education, 28(4), 526-534.

Martínez, R., Moreno, R., \& Muñiz, J. (2005). Construcción de los ítems. En J. Muñiz, A. Fidalgo, E. García Cueto, R. Martínez y R. Moreno (Eds.), 
Cuadernos de Estadística 30. Análisis de los items (pp. 9-52). Madrid: La Muralla.

Maurer, T. J. \& Pierce, H. R. (1998). A comparison of Likert scale and traditional measures of self-efficacy. Journal of Applied Psychology, 83(2), 324-329.

Pajares, F. (2006). Self-efficacy during childhood and adolescence. In F. Pajares y T. Urdan, (Eds.), Adolescence and education. Self-efficacy beliefs of adolescents, Vol. 5, (pp. 339-367). Greenwich, CT: Information Age Publishing.

Pas, E. T., Bradshaw, C. P., \& Hershfeldt, P. A. (2012). Teacher and school-level predictors of teacher efficacy and burnout: identifying potential areas for support. Journal of School Psychology, 50(1), 129-145.

Prieto, L. (2007). Autoeficacia del profesor universitario. Eficacia percibida y práctica docente. Madrid: Narcea.

Prieto, G. \& Delgado, A. (2010) Fiabilidad y validez. Papeles del Psicólogo, 31(1), 67-74.

Poulou, M. (2007). Personal teaching efficacy and its sources: student teacher's perceptions. Educational Psychology, 27(2), 191-218.

Robert, J.K., \& Henson, R.K. (2001, April). A confirmatory factor analysis of a new measure of teacher efficacy: Ohio State Teacher Efficacy Scale. Paper presented at the Annual Meeting of the American Educational Research Association, Seattle, WA.

Ross, J., \& Bruce, C. (2007). Professional development effects on teacher efficacy: results of randomized field trial. Journal of Educational Research, 101(1), 50-60.

Rotter, J. B. (1966). Generalized expectancies for internal versus external control of reinforcement. Psychological Monographs, 80, 1-28.

Sari, H., Çeliköz, N., \& Seçer, Z. (2009). An analysis of pre-school teachers' and student teachers' attitudes to inclusion and their self-efficacy. International Journal of Special Education, 24(3), 29-44.
Schermelleh-Engel, K., Moosbrugger, H., \& Müller, H. (2003). Evaluating the fit of structural equation models: Test of significance and descriptive goodness-of-fit measures. Methods of Psychological Research online, 8, 23-74.

Skaalvik, E. M. \& Skaalvik, S. (2007). Dimensions of teacher self-efficacy and relations with strain factors, perceived collective teacher efficacy, and teacher burnout. Journal of Educational Psychology, 99(3), 611-625.

Solís, M., Núñez, C., Contreras, I., Rittershaussen, S., Montecinos, C., \& Walker, H. (2011). Condiciones de la formación práctica de los futuros profesores. Estudios Pedagógicos, 37(1), 127-147.

Tagle, T., del Valle, R., \& Flores, L. (2012). Las creencias de autoeficacia percibida de estudiantes de pregrado de pedagogía en inglés. Revista Iberoamericana de Educación, 58(4), 1-12. Recuperado de http://www.rieoei.org/rie_contenedor.php?numero $=4667 \&$ titulo $=$ Las $\% 20$ creencias $\% 2$ de $\% 20$ autoeficacia\%20percibida\%20 de\%20estudiantes\%20de\%20pregrado\%20de\%20 pedagog\%EDa\%20en\%20ingl\%E9s

Tschannen-Moran, M., \& Johnson, D. (2011). Exploring literacy teachers' self-efficacy beliefs: potential sources at play. Teaching and Teacher Education, 27(4), 751-761.

Tschannen-Moran, M., \& Woolfolk, A. (2001). Teacher efficacy: capturing an elusive construct. Teaching and Teacher Education, 17(7), 783-805.

Tsigilis, N., Koustelios, A., \& Grammatikopoulos, V. (2010). Psychometric properties of the Teachers' Sense of Efficacy Scale within the Greek educational context. Journal of Psychoeducational Assessment, 28(2), 153-162.

Wolters, C. A., \& Daugherty, S. G. (2007). Goal Structures and Teachers' Sense of Efficacy: Their Relation and Association to Teaching Experience and Academic Level. Journal of Educational Psychology, 99(1), 181-193. 


\section{Apéndice 1: Escala Sentimiento de Autoeficacia en el Profesor}

Este cuestionario ha sido diseñado para que podamos comprender mejor qué aspectos generan dificultades en el quehacer pedagógico. Rogamos nos dé su opinión sobre cada una de las siguientes preguntas. Le pedimos por favor no deje ningún enunciado sin responder. Sus respuestas serán confidenciales.

INSTRUCCIONES: Conteste con una X según su preferencia en la columna correspondiente.

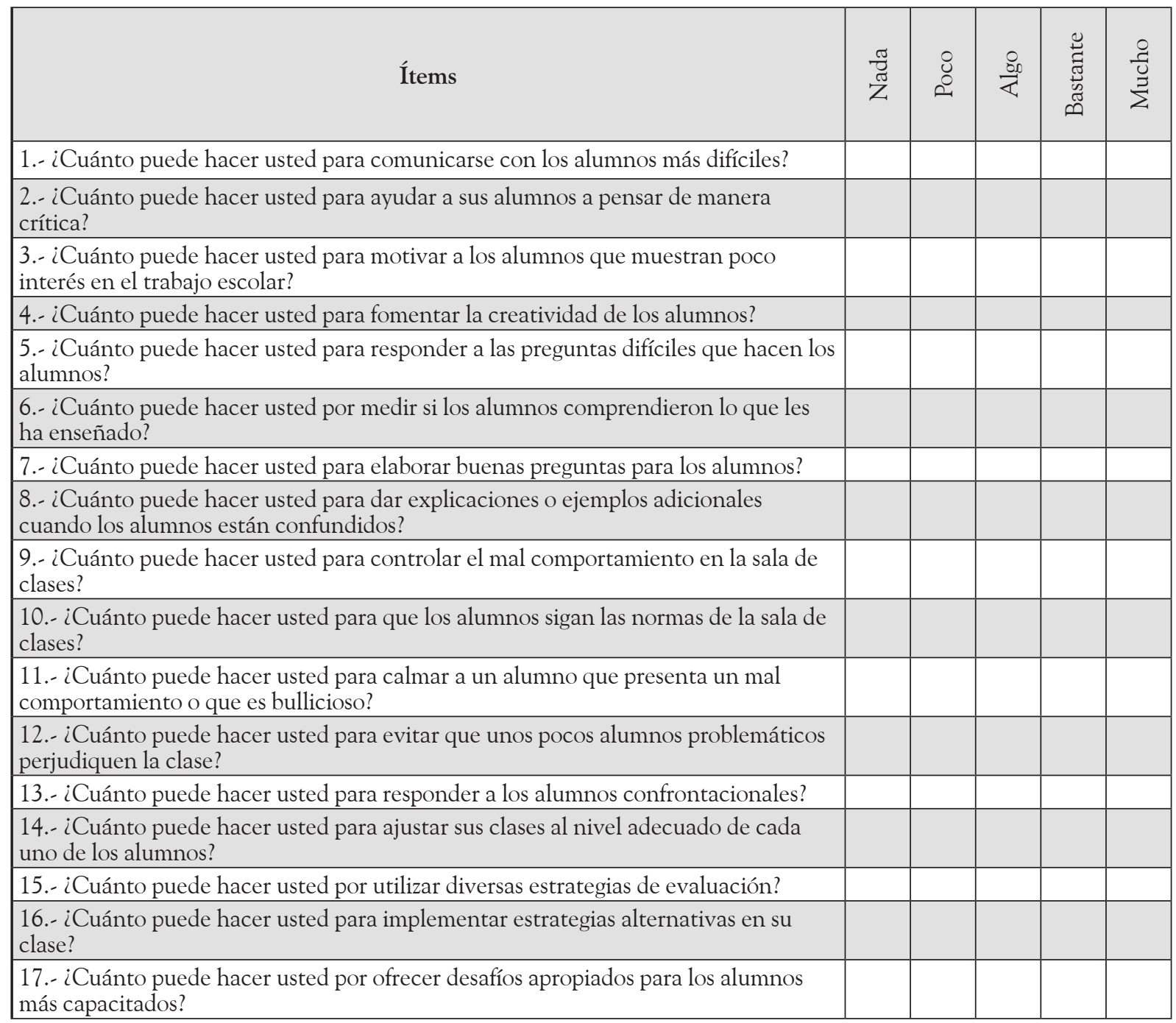


